\title{
The meson target stations and the high power spallation neutron source SINQ at PSI
}

\author{
D. Kiselev • P. Baumann • B. Blau • K. Geissmann • \\ D. Laube $\cdot$ T. Reiss $\cdot$ R. Sobbia $\cdot$ A. Strinning $\cdot$ \\ V. Talanov $\cdot$ M. Wohlmuther
}

Received: 10 September 2014/Published online: 28 February 2015

(c) Akadémiai Kiadó, Budapest, Hungary 2015

\begin{abstract}
The Paul Scherrer Institut operates two meson graphite targets, Target $\mathrm{M}$ and Target $\mathrm{E}$, for creating the world's most intense pion and muon beams by using $590 \mathrm{MeV}$ protons and c.w. beam currents of up to $2.4 \mathrm{~mA}$ $(=1.4 \mathrm{MW})$. The energy deposit on Target $\mathrm{E}$ is $20 \mathrm{~kW} \mathrm{~mA}{ }^{-1}$. The proton beam feeds also the spallation neutron source SINQ, which operates in DC mode and produces thermal and cold neutrons. The SINQ target consists of a bundle of lead filled Zircaloy tubes. In this report the continuous developments of both target facilities and their operation are presented.
\end{abstract}

Keywords High power target - Meson production · Spallation neutron source

\section{Introduction}

The Paul Scherrer Institut (PSI), the largest national research laboratory of Switzerland, operates the most intense muon source in the world as well as the only MW-class continuous spallation neutron source. At the six secondary beam lines of the two meson production stations, Target $\mathrm{M}$ and Target $\mathrm{E}$, surface muons in the energy range of $0.1-4 \mathrm{MeV}$, unique low energy muons (LEM) of $0.5-30 \mathrm{keV}$, high energy muons $(15-60 \mathrm{MeV})$ from the decay of pions in flight and pions as well are available. Surface muons are produced from decay of pions at rest in a tiny region up to $0.8 \mathrm{~mm}$ from the surface of the target made from graphite. The rate of positive

D. Kiselev $(\bowtie) \cdot$ P. Baumann · B. Blau · K. Geissmann .

D. Laube - T. Reiss $\cdot$ R. Sobbia $\cdot$ A. Strinning $\cdot$ V. Talanov

M. Wohlmuther

Paul Scherrer Institut PSI, 5232 Villigen, Switzerland

e-mail: Daniela.Kiselev@psi.ch surface muons on the muon moderator target of the LEM apparatus accounts to $1.7 \times 10^{8} \mu^{+} \mathrm{s}^{-1}$ at $2 \mathrm{~mA}$ protons on Target E. This corresponds to $1.9 \times 10^{7} \mu^{+} \mathrm{cm}^{-2} \mathrm{~s}^{-1}$ due to the contribution of muons from pion decays in flight, the muon beam is not $100 \%$ polarized but up to $95 \%$. They are for instance used for the investigation of the magnetic structure of materials.

The thermal neutron flux in SINQ is of the range of $10^{14} \mathrm{n} \mathrm{cm}^{-2} \mathrm{~s}^{-1}$ and is well competitive with modern medium flux research reactors. Besides thermal neutrons also cold neutrons are produced in a $25 \mathrm{~K} \mathrm{D}_{2}$ moderator. Neutrons are extracted through 6 beam ports, which are connected to several beam lines. A large area of application is the investigation of modern materials' atomic and magnetic structure and excitations with neutrons, which is complementary to the examination with muons or X-rays. Further radiography with cold and thermal neutrons allows for a wide range of investigations. As X-rays can only pass through light materials, the inside of a motor or $\mathrm{Pb}$ remains hidden. With the radiography station at SINQ the functioning of a motor in operation or the contents inside a lead can are observed with good contrast.

Details and ongoing developments of the two meson production target stations Target $\mathrm{E}$ and Target $\mathrm{M}$ as well as of the spallation neutron source, SINQ, will be described below. A second spallation source, the ultracold neutron source (UCN), which is operated in pulsed mode ( $1 \%$ duty cycle) since the end of 2010 at PSI, will not be discussed in this paper.

\section{The HIPA facility}

The meson target stations are fed by $590 \mathrm{MeV}$ protons with beam currents of up to $2.4 \mathrm{~mA}$ from the high intensity 
proton accelerator (HIPA), which delivers the most intense continuous proton beam in the world. An overview of HIPA is given in Fig. 1. The protons are produced in a compact electron cyclotron resonance (ECR) source [1] and gain $870 \mathrm{keV}$ by a Cockcroft-Walton. Afterwards they are accelerated in two cyclotrons called Injector2 and Ring to $72 \mathrm{MeV}$ and finally to $590 \mathrm{MeV}$, respectively. The beam first passes the meson production target station Target $\mathrm{M}$ with an effective thickness of $5 \mathrm{~mm}$ graphite, and then Target $\mathrm{E}$ with a thickness of $40 \mathrm{~mm}$. In Target $\mathrm{E}$ the protons lose $15 \mathrm{MeV}$ of their initial energy and $\sim 30 \%$ of the intensity, mainly due to multiple scattering and ionization in the meson production targets and the subsequent collimator system KHE0-KHE3. The collimator system is needed to limit the activation in the area and shape the beam for the operation of SINQ. In normal operation the beam is first bent downwards and afterwards upwards to hit the SINQ target from below. If SINQ is not ready to take beam, protons $(<1.7 \mathrm{~mA})$ are steered to a beam dump to keep the meson production target stations still in operation.

\section{The meson production target stations}

The main challenges for the meson production target stations are the high radiation levels and the power deposition. $590 \mathrm{MeV}$ protons of $2.4 \mathrm{~mA}$ deposit about $50 \mathrm{~kW}$ in the $40 \mathrm{~mm}$ graphite of Target $\mathrm{E}$. This requires an efficient cooling system and puts high demands on the target material. The material has to withstand high temperatures and has to be resistant against radiation damage, i.e. embrittlement and deformation. To distribute the power in the target, a wheel rotating with $1 \mathrm{~Hz}$ was chosen. This requires bearings able to survive the harsh irradiation environment. They will be described later.
Up to the 80 's beryllium was used as target as multiple scattering is low. The average beam current was on the order of $100 \mu \mathrm{A}$ or less. The targets failed at beam currents of 120 and $150 \mu \mathrm{A}$ for target diameters of 0.19 and $0.28 \mathrm{~m}$, respectively [2]. The target material was then replaced by pyrolytic graphite, which consists of thin sheets in its lattice structure. Therefore, the physical properties like thermal conductivity or thermal expansion are highly anisotropic. Under irradiation with proton currents of about $100 \mu \mathrm{A}$ for roughly 1 month the pyrolytic graphite showed swelling of about $10 \%$ [3]. Since then different grades of polycrystalline graphite from SGL-CARBON [4] are in use, which consists of a large number of small single crystallites, irregularly arranged in space. Therefore, physical properties are much more isotropic; small grain sizes further improve the isotropy. The physical properties also strongly depend on the graphite's production process, the temperature treatment and the raw material. As the graphite wheel of Target $\mathrm{E}$ is radiation cooled, irradiation induced changes of the thermal conductivity are unimportant for the operation of the target station. Since the rim of the wheel is only $6 \mathrm{~mm}$ wide, rim deformations of 1-2 $\mathrm{mm}$ along the wheel could cause a beam fraction $(\sigma \sim 1 \mathrm{~mm})$ to bypass the target. This would not only reduce the meson production rate but also leads to a pencil beam hitting the SINQ target window, which could not withstand such power densities. Although there are several safety systems, which would switch off the beam by fast interlock, this situation has to be avoided. It was observed that the radial deformation normalized to 1 Ah beam charge increases with the beam current (see Fig. 2). By changing the grade from R6300P to R6400P the radial deformation could be reduced by almost an order of magnitude. Since more than 10 years the Target $E$ thickness was reduced from 60 to $40 \mathrm{~mm}$, which obviously reduces the stability of the rim

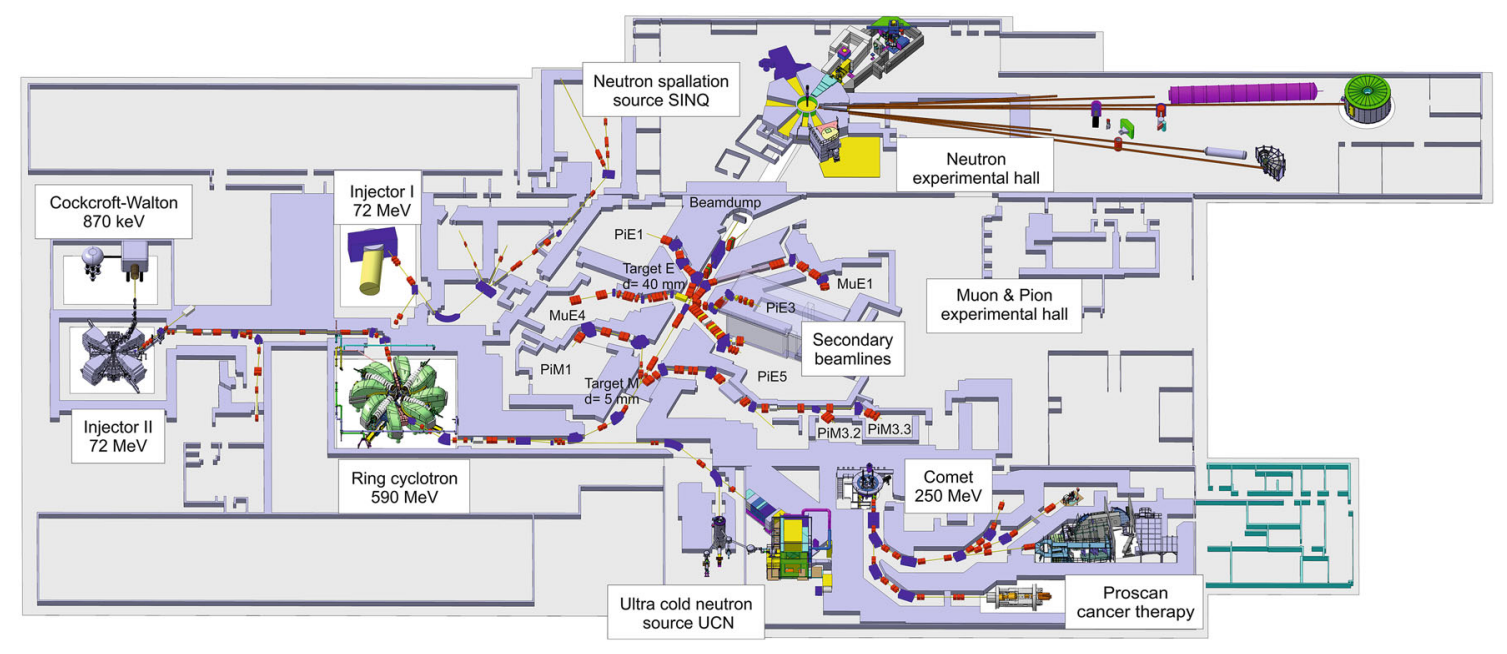

Fig. 1 Overview of the accelerator facility HIPA (courtesy of Th. Rauber, PSI) 


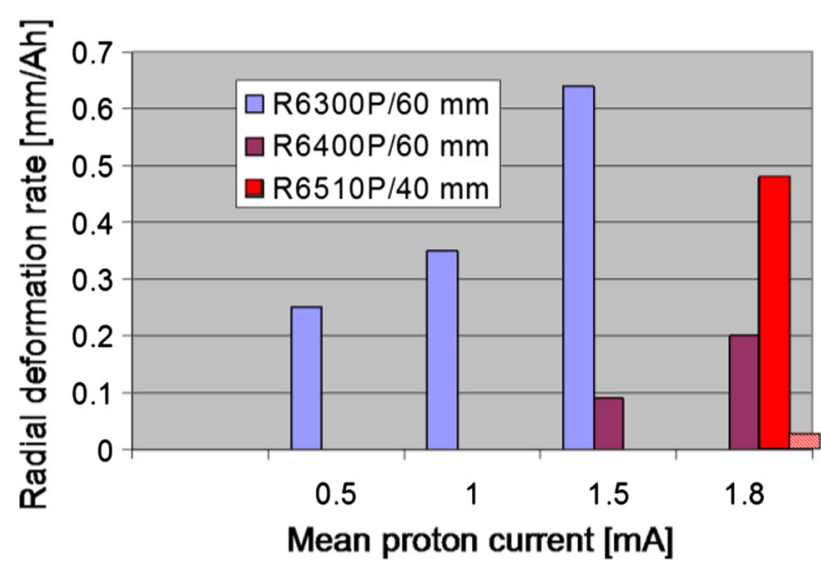

Fig. 2 Radial deformation of different types of graphite wheels for Target E

and therefore increases the radial deformation. The reason for the reduction in thickness is that the beam losses decrease from $40 \%$ for the $60 \mathrm{~mm}$ target to $30 \%$ for a $40 \mathrm{~mm}$ target, and therefore decreases the performance of the SINQ target. In addition, the current limit is $2 \mathrm{~mA}$ with $60 \mathrm{~mm}$ target due to the actual design of the KHE2\&3 system. The use of thinner targets increases the radial deformation rate by more than a factor 2 (see Fig. 2).

Therefore the design of the target was modified (see Fig. 3). The $40 \mathrm{~mm}$ wide rim of the target was divided into 12 segments. Between the segments a $1 \mathrm{~mm}$ wide gap allows for thermal expansions as well as dimensional changes due to irradiation. At the operational temperature of Target E, $1700 \mathrm{~K}$, the gap is only $0.5 \mathrm{~mm}$ wide and the intensity variation of the proton beam after passing the graphite wheel is negligible. So far the record in the highest integral beam current is held by Target E93, which was irradiated with 33.5 Ah. This corresponds to an operational period of about 3.5 years and an irradiation damage rate of 3.4 DPA. By visual inspection it was observed that the shapes of the segments were distorted in such a way that one corner pointed upward with the two neighboring ones

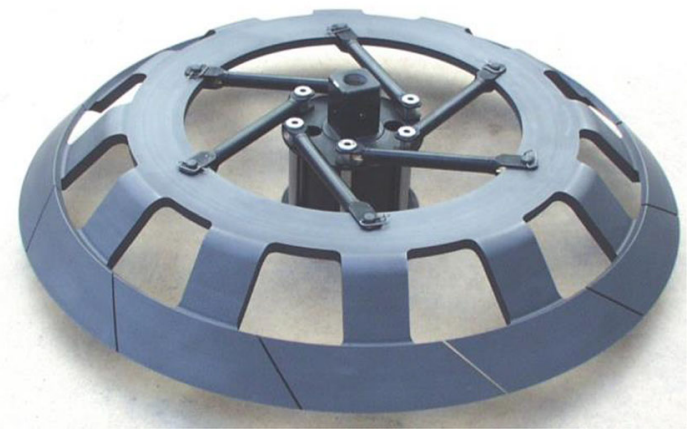

Fig. 3 New and actual design of the graphite wheel in the Target E station pointing downward. From the photo (see Fig. 4) it is difficult to quantify, how much the segments are tilted against each other. For this a device consisting of three dial gauges was constructed, measuring the displacement between the corners of two adjacent sections. The device was tested using a non-irradiated graphite wheel. The measurement at the irradiated Target E93 will be performed soon. For the future ANSYS [5] calculations are planned optimizing the shape further with the aim to reduce the thermal stress in the rim of the graphite wheel.

The most critical parts of Target $\mathrm{E}$ are the ball bearings. Due to the high irradiation no grease can be used for the bearings. Grease would get hard and brittle very fast and the bearing would stick. At Target $\mathrm{E}$ two radial deep grove ball bearings are mounted along the rotation axis of the wheel and one angular contact ball bearing is located at the bottom of the $2 \mathrm{~m}$ long drive shaft. On the upper end of the drive shaft the motor is mounted, far away from the high radiation area. The life time of the commercially available motor is 5-8 years. At $2 \mathrm{~mA}$ the bearing closest to the graphite wheel has a temperature of about $140{ }^{\circ} \mathrm{C}$ at the inner side of the inner ring, the second $50{ }^{\circ} \mathrm{C}$. The graphite wheel with its operation temperature of about $1700 \mathrm{~K}$ at $2 \mathrm{~mA}$ is cooled by radiation only, which is due to the large emissivity of graphite. The local shielding, close to the target wheel, made from copper and actively cooled with water, absorbs the heat radiated off the target.

The first sets of bearings used were fabricated from stainless steel. The inner parts were coated with silver, which served as lubricant. For low proton current $(\sim 0.5 \mathrm{~mA})$ one set of bearings lasted for one operational year with about $5000 \mathrm{~h}$. When the proton beam increases to about $1 \mathrm{~mA}$, their life time dropped to $1000 \mathrm{~h}$. Although a complete exchange of Target E using the remotely controlled and shielded exchange flask takes only 2 days, a large number of personnel is involved. Therefore, new kinds of bearings were tried out and tested. This search ended with hybrid bearings delivered by the company GMN in Bonn, Germany. The balls are fabricated from $\mathrm{Si}_{3} \mathrm{~N}_{4}$, a black ceramic. The other parts are of stainless steel. The inner and outer rings are coated with a mix of $\mathrm{MoS}_{2}$ and silver, which serves as a lubricant. Since $\mathrm{MoS}_{2}$ can chemically change to $\mathrm{MoS}_{3}$ in air,

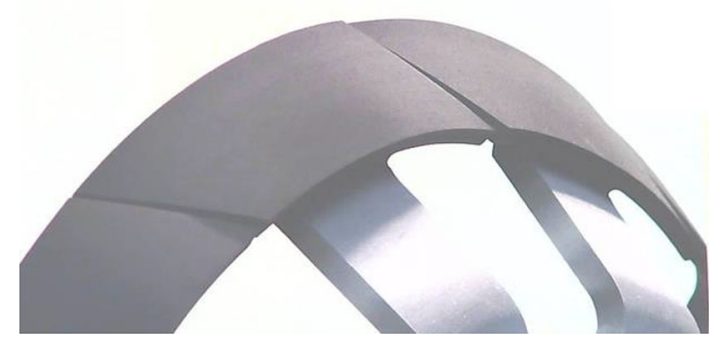

Fig. 4 Distorted segments of Target E93 
the coating would get brittle. Therefore, the bearings are stored in vacuum. Their life time varies between half and a full operational year, much less than the life time of the graphite wheel. Therefore, new bearings are currently being tested. At the moment bearings from HWG [6] and CEROBEAR [7], both located in Germany, are under long time testing. The ones from HWG are completely made from $\mathrm{Si}_{3} \mathrm{~N}_{4}$ whereas the ones from CEROBEAR have the cage fabricated from stainless steel. The bearings are tested under conditions which as closest as possible resemble real conditions, however without radiation. In the newly built test stand two graphite wheels are rotating at the same time completely independent from each other. The inner ring of the front deep grove bearing is heated to $140{ }^{\circ} \mathrm{C}$ to simulate the heating by the proton beam. The current of the motor is monitored and logged in the same way as for the Target $\mathrm{E}$ station. An increase of the motor current indicates that at least one of the three bearings is scratching. One set of bearings without coating from CEROBEAR was in use for 1 year without any visible damage. Another set of bearings from CEROBEAR is coated with silver but not yet tested. The three sets of HWG bearings tested failed all after 2-3 weeks already. At a first glance it seems that the clearance between the cage and the rings are not sufficient. When in operation there is always some abrasive wear from the drive shaft or gear wheels. Dust and small particles are transported into the bearing, which finally leads to a failure of the bearings.

The failure of bearings due to abrasive wear is not only restricted to the test stand but an even more serious problem at the Target E station, which is in use since 1990. The bearing most affected is the angular contact ball bearing, which is mounted in a vertical position close to the gear wheels. In the last years it was observed that the angular contact ball bearing fails more often than the two deep groove ball bearings, although the radiation level and temperature is the highest at the front deep groove ball bearing. As it was speculated that dirt from abrasive wear could cause the problem, the insert for the drive shaft was investigated with a camera. The camera had to be quite small as the diameter of the insert is $3.8 \mathrm{~cm}$, remotely controlled, protected against contamination and equipped with light. These requirements were matched by a commercial camera used by chimney sweepers. A movie of the inspection was recorded. On the seat of the angular contact ball bearing a few locations were covered with dust. A special tool was constructed to remove the dust. The procedure had to be repeated two times until the dust was removed. Future operation of the Target $\mathrm{E}$ station will show whether the life time of the angular contact bearing could be increased.

The life time of the bearings in the Target $M$ target station is much longer. One set is usually in operation for several years. The reason is that in the graphite wheel with an effective target thickness of $5.2 \mathrm{~mm}$ less radiation is produced. Only a fraction of $1.6 \%$ from the proton beam is lost. Furthermore the first bearing of Target $\mathrm{M}$ is not directly located at the graphite wheel but about $48 \mathrm{~cm}$ upstream, deep in the shielding. The design of the graphite wheel of Target $\mathrm{M}$ is simpler compared to Target E. It consists of a round plate with a $20 \mathrm{~mm}$ rim, which rotates with $1 \mathrm{~Hz}$. The life time record is $50,000 \mathrm{~h}$ up to now, which corresponds to $44 \mathrm{Ah}$. Due to the lower operation temperature of $1100 \mathrm{~K}$ at $2 \mathrm{~mA}$ the graphite wheel is mainly cooled by conduction and not by thermal radiation.

In case of a failure of one of the meson production targets, the target insert has to be removed with its shielding plug using a remotely controlled exchange flask due to the high dose rates of $1-3 \mathrm{~Sv} \mathrm{~h}^{-1}$ at the graphite wheel and bearing case. The exchange flask of Target $\mathrm{E}$ is shielded by $40 \mathrm{~cm}$ steel and has a net weight of $42 \mathrm{t}$. Target $\mathrm{E}$ is to removed vertically whereas the Target $\mathrm{M}$ is taken out in a horizontally. To access each target station first several meters of concrete have to be removed by crane.

\section{The spallation neutron source SINQ}

To produce neutrons from high energetic protons targets from heavy nuclei are preferred, which have naturally an excess of neutrons. The reaction process is called spallation. The energy of the primary particle, in case of SINQ $575 \mathrm{MeV}$ protons, is distributed amongst the nucleons of the heavy nuclei- $\mathrm{Pb}$ for SINQ. During this stage high energetic particle can be emitted. The nucleus is then left in an excited state and releases its excess energy by emitting light particles like neutrons, protons, deuterons, alphas and others. The neutrons from so called evaporation stage have an energy spectrum peaked around $1-2 \mathrm{MeV}$. This energy is too high to be used for neutron scattering experiments. Therefore, the neutrons are slowed down by moderators at different temperatures to obtain neutrons of equivalent thermal energies. The target is surrounded by a large vessel of $\mathrm{D}_{2} \mathrm{O}$ at ambient temperature, where neutrons are thermalized to about $0.025 \mathrm{eV}$ (thermal neutrons). To further cool the neutrons down, a cold moderator of 201 liquid $\mathrm{D}_{2}$ at $25 \mathrm{~K}$ is installed in SINQ. Neutrons below $5 \mathrm{meV}$ are called cold. At SINQ 12 neutron scattering instruments (diffractometers, reflectometers, spectrometers, small angle scattering instruments) are viewing the cold moderator whereas four instruments use thermal neutrons. Main application is material research and the investigation of biological substances. To investigate the magnetic structure of materials in details neutrons may be polarized by supermirrors. Two beamlines are used for radiography with cold and thermal neutrons, respectively. Besides the experimental stations for neutron scattering and radiography there are three different possibilities to irradiate 
materials, e.g. for testing its irradiation hardness or for isotope production. Two irradiation stations, called PNA and NAA, are located 40 and $80 \mathrm{~cm}$ from the central target. Small samples are packed into a capsule and transported via a rabbit system to the irradiation station. At PNA the sample is irradiated with $4 \times 10^{13} \mathrm{n} \mathrm{cm}^{-2} \mathrm{~s}^{-1}$ for a maximum irradiation time of $1000 \mathrm{~h}$. The third possibility is the SINQ Target Irradiation Program (STIP). Here a tube of about $10 \mathrm{~mm}$ diameter and length of about $120 \mathrm{~mm}$ is loaded with samples and mounted in the SINQ target. Obviously, this can only be done, when a new target is built and inserted. Every 2 years the SINQ target is exchanged, stored in the target storage area for at least 1 year and then disassembled. This means that the irradiation time is fixed to 2 years and it takes approximately 4 years counting from the sample preparation until the sample take-out. The STIP samples are usually already in the shape to be used for mechanical material tests.

The target insert is a cylindrical structure of about $5 \mathrm{~m}$, which is mounted in a vertically up-right position. The lower part with the target containing $\mathrm{Pb}$ has a diameter of $20 \mathrm{~cm}$, whereas the upper part, which serves as shielding, is about $40 \mathrm{~cm}$ in diameter. The lower part is surrounded by a double-walled safety shroud made from $\mathrm{AlMg}_{3}$, which contains the proton beam entrance window at the lower end. Between the two walls the shroud and the proton beam window are cooled by heavy water. Due to the high power irradiation by protons the target insert is exchanged every 2 years. After 2 years the beam entrance window suffered already from 20 to 30 DPA. Similar to Target E the SINQ target insert is removed with a shielded exchange flask and first transported to the target storage. In the storage area it is cooled by air and-if necessary-can be cooled by water. After 1 year it is disassembled in the hot cell for disposal as radioactive waste.

Since the $575 \mathrm{MeV}$ proton beam is fully stopped in the target, a large amount of energy is deposit in the target, which requires an efficient cooling. The target itself contains about 300 separate tubes filled with lead (called Cannelloni), which are cooled by heavy water at an average pressure of 7 bar. The tubes in the first row are empty. Their purpose is to make the water flow turbulent. This ensures that the hot water streaming at the tubes is mixed with colder water streaming in between the tubes. $\mathrm{D}_{2} \mathrm{O}$ is used instead of natural water, because of the lower neutron absorption cross section of D-H (factor 650). To distribute the heat deposition further, the beam is not pencil like but has an approximately double Gaussian shape with standard deviations of 2 and $3 \mathrm{~cm}$. The spread of the beam is caused mainly by multiple scattering at Target $\mathrm{E}$ and its elliptical shape is determined by the aperture of the collimator system KHE2\&3, which is located $4.7 \mathrm{~m}$ downstream of Target E. The Gaussian intensity distribution has a peak current density of $47.4 \mu \mathrm{A} \mathrm{cm}^{-2}$ at $2 \mathrm{~mA}$ [8]. From the point of energy/heat deposition and the subsequent thermal stress on the tubes this is not ideal. The central part of the tubes is much hotter and the $\mathrm{Pb}$ is partially molten. As it was shown by ANSYS simulation this leads to additional localized mechanical stress. Therefore, a conceptual study for producing a flat beam intensity profile was performed [9]. To monitor the heat deposition by the beam, some of the Cannellonis and a few other locations inside the target are equipped with thermocouples. The heat deposition is calculated by Monte Carlo particle transport programs like MCNPX [10] and fed to ANSYS [5] for simulating and optimizing the flow distribution and target cooling. Since the ANSYS modeling is quite complex, a calculation with a full 3D model including the partial melting of the lead is not yet available but under development.

The SINQ target station went into operation in 1996. The first target type consisted of a rod bundle of solid Zircaloy- 2 rods. The reason was that the behavior of Zircaloy under a high energetic proton bombardment had to be tested first, even though its good radiation resistance in thermal neutron fields is well known from its use as fuel rod cladding. One main difference is that due to the spallation process as well as due to disintegration of the heavy water, a larger amount of hydrogen isotopes is produced compared to reactors. Hydrogen can lead to embrittlement of Zircaloy by the formation of Zircaloy-Hydride. Compared to steel Zircaloy is the preferred material, because of its lower absorption cross section for thermal neutrons. The second target type MARK II was made from steel tubes filled with $\mathrm{Pb}$ already. In the last MARK II target a few Zircaloy tubes were inserted off-center, where the heat deposition was lower, since the influence of $\mathrm{Pb}$ onto the Zircaloy tube under irradiation had to be tested. This target achieved already $42 \%$ more neutron flux than the full Zircaloy target. Three targets of this kind (MARK II) were in use from 2000 to 2005. In 2006 a special target was in operation for 4 months. The so called MEGAPIE collaboration, which is a joint international initiative to demonstrate that a liquid metal target using lead-bismuth eutectic (LBE) can be successfully build, licensed and operated with $1 \mathrm{MW}$ proton beam power [11]. This initiative was mainly driven to demonstrate that the liquid metal target concept for accelerator driven systems (ADS) is feasible. Future ADS might be used for the transmutation of spent fuel in such a way that long lived radioactive isotopes are burned and consequently the final radioactive waste will decay to normal background levels considerably faster. In Europe the test of this concept is planned in Belgium (MYRRHA project) [12]. Compared to SINQ target MARK II used before the MEGAPIE showed an increase in the neutronic performance by $80 \%$ due to the use of pure heavy metal without additional cooling fluid or 

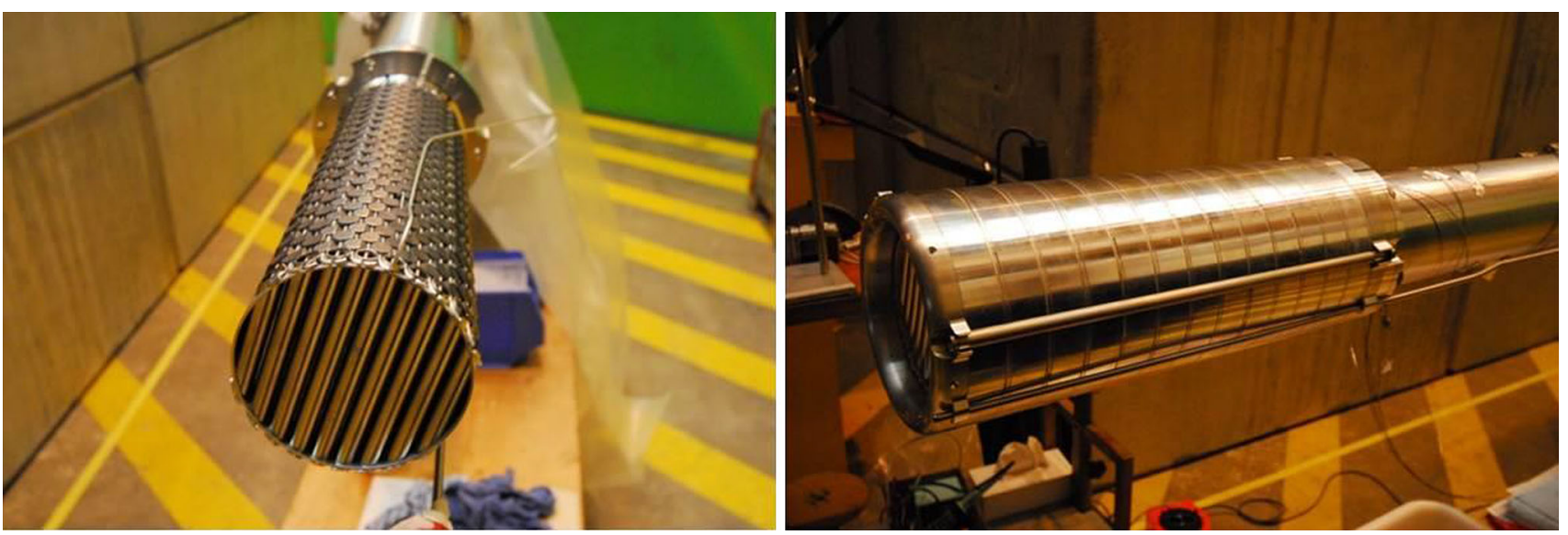

Fig. 5 Actually used SINQ target design MARK IV. Left Rods mounted in target holder. Right Target surrounded by Pb blanket

cladding. However, the effort to manufacture and operate the LBE target is much larger than for the solid SINQ targets. In addition, the operation is more risky and costly. In case of a leak highly radioactive liquid metal would end up in a container in the SINQ basement and operation of the source would be discontinued for at least a year. Therefore, at PSI it was decided to cease manufacturing and development of liquid metal targets but instead to further improve the performance of solid targets. As a first step the solid target following MEGAPIE was equipped with tubes from Zircaloy solely. This lead to an increase in neutron flux of $10 \%$ compared to the MARK II target operated before MEGAPIE. Major modifications with the aim to further increase the performance of the solid target were introduced in MARK IV 2009. The Monte Carlo particle transport program MCNPX was used to optimize the target setup; it was shown that a round cross section of the target is more advantageous than the rectangular in MARK II and III. To increase the Pb content in the target the Cannellonis were closer packed. In addition, the proton beam entrance window was inverted. The benefit is that the protons loose less energy in the water. An increased neutron flux of $10 \%$ was predicted by the beam entrance window inversion. Further, a layer of $2.2 \mathrm{~cm} \mathrm{~Pb}$ was introduced around the rod bundle (see Fig. 5). This layer, called $\mathrm{Pb}$ blanket, works as a reflector for neutrons moderated in the $\mathrm{D}_{2} \mathrm{O}$ vessel. The second purpose of the blanket is to minimize the moderation of neutrons inside the target. As a consequence, the thermal neutron flux maximum is 'pushed' outwards closer to the neutron beam ports and to the moderator inserts. According to the MCNPX predictions all modifications together increased the neutron flux by $40 \%$ compared to the MARK III target series. This is only $15-20 \%$ less than the LBE target MEGAPIE and therefore justifies the decision to cease the liquid metal target efforts. The solid target type MARK IV is still in

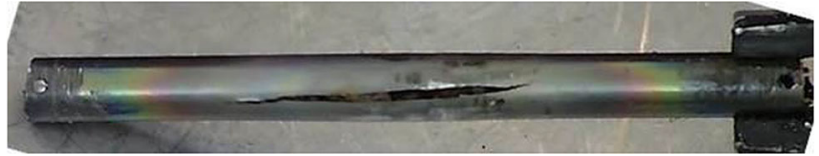

Fig. 6 Broken tube from row 16 of Target 8

operation up to now. In Table 1 there is a summary of all SINQ targets so far in use with a short description of them.

\section{Challenges and future developments}

One problem that needs further attention is the occasional cracking of tubes during operation, which occurred for a $\mathrm{Pb}$ filled tube in Target 8 (2009-2010) in row 16 for unknown reasons (see Fig. 6). This causes contamination of the $\mathrm{D}_{2} \mathrm{O}$ cooling water with radioactive isotopes. The isotopes were removed by the filters installed in the water cooling system. In the last target investigated, Target 9, a Zircaloy tube filled with STIP samples broke, when it was taken out from the target holder. An empty tube and one filled with $\mathrm{Pb}$ were extracted for further post irradiation investigations in addition. Crack propagation tests and examination of the $\mathrm{H}$-content are planned.

Since 2010 the tubes suffer from additional thermal stress. The beam is now switched to the UCN target station for about $4 \mathrm{~s}$ every $\sim 8 \mathrm{~min}$. Since the SINQ target rods cool down in about $2 \mathrm{~s}$, this causes 50,000 thermal cycles per year and can lead to fatigue.

In future SINQ targets the wall thickness of the Zircaloy tubes will be reduced from 0.75 to $0.606 \mathrm{~mm}$ and at the same time the outer diameter is reduced. This allows on one hand for better cooling but also more rods in the target keeping the pitch constant. Therefore, the amount of $\mathrm{Pb}$ in the target will be increased. In addition, Monte Carlo 
Table 1 Different kind of SINQ targets

\begin{tabular}{llll}
\hline & In use from to & Short description & Cross section \\
\hline MARK I & $1996-1999$ & Solid Zircaloy rods & Hexagonal \\
MARK II & $2000-2005$ & Pb in stainless steel tubes & Quadratic \\
MEGAPIE & 2006 & Liquid metal LBE & Round \\
MARK III & $2007-2008$ & Pb in Zircaloy tubes & Quadratic \\
MARK IV & 2009 & Pb in Zircaloy tubes with Pb blanket & Round \\
\hline
\end{tabular}

particle transport calculations already started to optimize and redesign the cold and thermal moderator inserts and to study upgrades of other parts such as the neutron guides.

\section{Summary}

The meson production and neutron spallation target stations have been working reliable without major problems for more than 20 years. The crucial parts of the meson production Target $\mathrm{E}$ are the bearings, which suffer from the increasing proton current and the higher radiation levels over the past years. New bearing types are currently being tested with the goal to prolong their overall life time in the harsh environment around Target E.

The neutron flux in SINQ could be increased by a factor of 2.19 compared with the first Zircaloy solid target. In the same period the proton current increased in addition by a factor of 1.75 , i.e. a total gain factor of 3.8 in the neutron flux at the instruments was achieved. In the SINQ Target 8 and 9 damaged Zircaloy tubes were found. The reason is not known yet. Two tubes from Target 9 will be investigated further.
Acknowledgments We would like to thank Kurt Clausen and Mike Seidel for valuable advices, the financial support and the faith in our work. Further we thank the SINQ operation group for maintaining the SINQ target station and last but not least the service groups for the good cooperation, when e.g. exchanging the targets.

\section{References}

1. Baumgarten C, Barchetti A, Einenkel H, Goetz D, Schmelzbach PA (2011) Rev Sci Instrum 82:053304

2. Heidenreich G (2002) Proceedings of the 20th international workshop of high intensity and high brightness hadron beams, Batavia, Illinois, USA. AIP Conf Proc 642:122-124

3. Heidenreich G, private communication

4. SGL-CARBON, http://www.sglgroup.com. Accessed 19 Feb 2015

5. ANSYS Inc. http://www.ansys.com/. Accessed 10 Sept 2014

6. HWG, 71272 Renningen, Germany

7. CEROBEAR, 52134 Herzogenrath, Germany

8. Reggiani D, Reiss T, Seidel M, Talanov V, Wohlmuther M (2014), submitted to Phys Rev ST-Acc \& Beams

9. Reggiani D, Kiselev D, Reiss T, Sobbia R, Talanov V, Wohlmuther M (2014). In: Proceedings of the 5th international part ACC conference, Dresden, Germany

10. Pelowitz DB (2011), MCNPX User's Manual Version 2.7.0, Los Alamos National Laboratory Report LA-CP-11-00438

11. Wagner W, Groeschel F, Thomsen K, Heyck H (2008) J Nucl Mater 377:12-16

12. MYRRHA project. http://myrrha.sckcen.be/. Accessed 10 Sept 2014 